\title{
Naringenin-Loaded TPGS Polymeric Nanosuspension: In-Vitro and In- Vivo Anti-Inflammatory Activity
}

\author{
Sumathi Rajamani, Tamizharasi Sengodan, Sivakumar Thangavelu, Nikhitha K Shanmukhan and \\ Arun Radhakrishnan*
}

Department of pharmaceutics, JSS College of pharmacy, Ooty JSS Academy of higher education and research India. Near Rose garden, Ooty, Tamil Nadu 643001, India

Info Article
Submitted: 30-03-2019
Revised: 27-05-2019
Accepted: $24-09-2019$
*Corresponding author
Arun Radhakrishnan
Email:
arunpharma93@gmail.com

Info Article

Revised: 27-05-2019

Accepted: 24-09-2019

*Corresponding autho

Email:

arenpharma93@gmail.com

\begin{abstract}
Naringenin, (NAR) from Citrus grandis (L.) Osbeck, family Rutaceae, exhibit extensive pharmacological action, lacks significance in application due to low aqueous solubility approximately $0.214 \mathrm{mg} / \mathrm{mL}$, which results in low bioavailability (5.8\%). Nanosuspension of NAR (NARNS) was prepared with various concentrations of polymers by high pressure homogenization technique. Physicochemical properties of the formulations were studied and optimized in our previous studies. The present study was performed further to identify the anti-inflammatory activity of the NARNS formulation in comparison with standard drug and NAR. Denaturation of protein and membrane stabilization methods were chosen for in-vitro evaluation and invivo studies performed were acute inflammatory studies (carrageenaninduced paw edema) and chronic inflammatory studies (cotton pellet granuloma) on Wistar albino rats. The optimum concentration of stabilizer and co-stabilizer chosen for this study was 1:1.5:1 with ps $80.52 \pm 0.13$ with better solubility when compared to NAR. The studies demonstrated significantly greater anti-inflammatory activity of NARNS compared to NAR and the standard drug at a lower concentration.
\end{abstract}

Keywords: oxidative stress, anti-inflammatory, Naringenin

\section{INTRODUCTION}

Flavonoids are a group of polyphenolic compounds, which are distributed throughout the plant kingdom. Flavonoids possess several pharmacological activities, such as antioxidant, anti-allergic, anti-bacterial, anti-inflammatory, anti-mutagenic and anti-cancer activity. NAR $(4,5$, 7-trihydroxy flavanone), a flavone, present extensively in grapefruit, citrus fruits, and tomato skin. NAR possess a wide range of pharmacological activities such as anti inflammatory activity, antibacterial activity, anti-hyperlipidemic, antioxidant, antiplatelet activity, cardioprotective, hepatoprotective, anticonvulsant and anticancer activity (Ferrero-Miliani L., 2007; William, 1989; Wolfe et al., 1999; Ameer,1996; Cavia-Saiz, 2010, Choi, 1991; Dou, 2009.) Poor bioavailability resulted from solubility, instability in physiological medium and extensive hepatic metabolism hamper the therapeutic applications of NAR (Yen, 2008; Wen, 2010). Nanosuspension is one of the preferable dosage forms to overcome the issues of drug degradation, solubility, and to deliver preferable biological drug concentrations (Jain, 2011). In our former studies, high-pressure homogenization method was employed to prepare NARNS from Soya lecithin, Polaxamer-407, Polaxamer-188, Hydroxy propyl methyl cellulose (HPMC) and Tween-80 with D- $\alpha$-Tocopheryl polyethylene glycol succinate 1000 (TPGS). All the prepared formulations were in nano size. The stabilizer concentration represented better solubility when compared to NAR was 1:1.5:1 with a particle size $80.52 \pm 0.13 \mathrm{~nm}$. The solubility and dissolution of NAR in the form of NARNS were significantly higher than those of pure NAR (Sumathi, 2017). Anti-inflammatory activity of NARNS was evaluated in the current study.

\section{MATERIALS AND METHODS Chemicals}

NAR was purchased from Zim laboratories Limited, Nagpur, India. Soya lecithin was procured from Glenmark generics limited, Mumbai, India. TPGS was obtained from Ludwigshafen, Germany 
and used as received. Carrageenan from Sigma chemical and Co, Diclofenac sodium from SigmaAldrich and Sodium chloride from Merck were used in the study. All reagents and solvents used were of analytical grade. Vernier calipers used for the measurement of paw diameter were procured from Precision India Ltd, Mumbai.

\section{Animals}

Male albino Wistar rats (150-200g BW) were chosen for the study. The rats were obtained from College of Veterinary and Animal Sciences, Thrissur, India. Animal study protocol was approved by the Institutional Animal Ethics Committee (NCP/IAEC/2015-201604). The animals were maintained under specified standard laboratory conditions. (temperature was maintained at $25 \pm 2^{\circ} \mathrm{C}$ and relative humidity was $55 \pm 5 \%$. Dark/light cycle $(14 / 10 \mathrm{~h})$ was also maintained during the study period. The animals were allowed free access to a standard dry pellet diet and water ad libitum.

\section{In vitro anti-inflammatory activity Denaturation of the protein albumin}

The NAR, NARNS and the standard drug diclofenac sodium were studied for their anti-inflammatory activity (in vitro) by heat induced protein denaturation method. Different concentrations of NAR, NARNS and diclofenac sodium were prepared in $10 \mu \mathrm{g} / \mathrm{mL}, 20 \mu \mathrm{g} / \mathrm{mL}$, $40 \mu \mathrm{g} / \mathrm{mL}, 80 \mu \mathrm{g} / \mathrm{mL}$ and $160 \mu \mathrm{g} / \mathrm{mL}$. The reaction mixture (r) consists of different concentrations of NAR/NARNS/diclofenac (2mL) and $\mathrm{pH} 6.4$ phosphate buffer saline $(2.8 \mathrm{~mL})$ were mixed with egg albumin $(2 \mathrm{~mL})$ taken from fresh hen's egg. The mixture was then subjected to incubation for $15 \mathrm{~min}$ at $32^{\circ} \mathrm{C}$. After incubation, denaturation was resulted by increasing the temperature of the reaction mixture up to $70^{\circ} \mathrm{C}$ for $15 \mathrm{~min}$. Absorbance of the reaction mixture was measured once it reached the room temperature at $660 \mathrm{~nm}$ and considering distilled water as blank. The trials were performed in triplicate for NAR, NARNS and control (diclofenac) (C). By applying the following formula, percentage inhibition for denaturation of protein was calculated (Gambhire, 2008; Alhakmani, 2013, 2014; Elias G., 1988).

$\%$ inhibition $=A B_{C}-\frac{B_{t}}{B_{C}} \times 100$

Where $\mathrm{AB}_{\mathrm{t}}=$ Absorbance oft, $\mathrm{AB}_{\mathrm{c}}=$ Absorbance of $\mathrm{C}$
The $\mathrm{IC}_{50}$ value was calculated from the percentage inhibition value using the below mentioned formula (Mizushima, 1986).

$$
I C_{50}=\left[\left(\frac{\sum C}{\sum I} x 50\right)\right]
$$

Where the $\sum \mathrm{C}=$ sum of extract concentrations used for testing; $\sum \mathrm{I}=$ the sum of the percentage of inhibition at different concentrations

\section{Membrane stabilization method}

A mixture of collected sheep blood and sterilized Alsevers solution (saline solution containing dextrose (2\%), $0.8 \%$ sodium citrate, $0.42 \% \mathrm{NaCl}$ and $0.055 \%$ citric acid - prevent coagulation of blood,) was used to perform the study. The mixture was ensured with separation of packed cells by centrifugation at 3000rpm. Isosaline solution was used to wash the separated packed cells after separation. A $10 \% \mathrm{v} / \mathrm{v}$ suspension of the cells was prepared using isosaline solution and this was studied for antiinflammatory property. Different concentrations of NAR, NARNS and the standard drug (diclofenac sodium) ( $2 \mathrm{~mL}$ ) were individually mixed in $2 \mathrm{~mL}$ of hyposaline, $1 \mathrm{~mL}$ of phosphate buffer, and $0.5 \mathrm{~mL}$ of RBC suspension. Samples were subjected to incubated at $37^{\circ} \mathrm{C}$ for $30 \mathrm{~min}$. The hemoglobin content was estimated followed by decantation of the supernatant liquid after centrifugation at $3000 \mathrm{rpm}$. The control was prepared by the same procedure, but the drug was replaced with equal volume of distilled water. The samples were analyzed spectrophotometrically at $560 \mathrm{~nm}$. The experiment was carried out in triplicate. Hemolysis produced in the control was considered $100 \%$ to calculate the \% hemolysis resulted in samples (Gandhidasan, 1991; Varadarasu, 2007; James, 2009). IC50 value was calculated according to the formula mentioned in the inhibition of protein albumin method.

$\%$ inhibition $=A_{C}-\frac{A_{t}}{A_{C}} \times 100$

Where $A_{t}=$ Absorbance of NAR/NARNS/Diclofenac sodium; $A_{c}=$ Absorbance of Control

\section{In vivo Anti-inflammatory activity}

The NAR and NARNS concentrations with maximum in vitro anti-inflammatory activity were subjected for in vivo anti-inflammatory activity study by carrageen induced paw edema and cotton pellet induced granuloma method. 


\section{Carrageenan-induced paw edema method}

Four groups of Wistar rats, each containing 6 animals of average weight were used for study. Group I was negative control which received distilled water, Group II serves as a positive control was administered with $5 \mathrm{mg} / \mathrm{kg}$ PO diclofenac sodium. Diclofenac sodium was the preferred positive control in studies to compare the results of the test with the known anti-inflammatory activity of the drug and to control the presence of unknown variables in the study. Group III and IV received NAR and NARNS respectively at the dose of 50 $\mathrm{mg} / \mathrm{kg}$ PO. Post 1 hour the paw edema was induced on the left hind paw of the rats by injecting $1 \mathrm{~mL}$ of $1 \% \mathrm{w} / \mathrm{v}$ of carrageen in saline solution into the subplantar tissues. After carrageenan induction, the paw perimeter of the rats was measured at hourly intervals for $3 \mathrm{~h}$ by using Verniercalipers. The right hind paw of the rats served as a normal which was not inflamed, the paw perimeter was compared with the standard group (Diclofenac sodium) for evaluation of anti-inflammatory activity (Jayaraman, 2012; Winter, 1962; Sarkhel., 2016: Ntandou, 2010). The percentage inhibition of antiinflammatory activity was calculated by the following formula.

$\%$ inhibition $=\frac{T_{c}-T_{c}}{T_{C}} \times 100$

$\mathrm{T}_{\mathrm{t}}=$ Thickness of paw perimeter in Test; $\mathrm{T}_{\mathrm{c}}=$ Thickness of paw perimeter in Control

\section{Cotton pellet-induced granuloma method}

Animals (Wistar rats) were divided into four groups of six rats in each. Adsorbent Cotton wool pellets weighing $10 \pm 1 \mathrm{mg}$ was prepared and sterilized using a hot air oven maintained at $120^{\circ} \mathrm{C}$. Sterilized cotton pellets were implanted subcutaneously into both sides of the groin region in rats followed by cleaning the abdomen area by shaving and swabbing ( $70 \%$ ethanol). Four groups of animals were treated (once daily) with NAR and NARNS $(50 \mathrm{mg} / \mathrm{kg}$ PO), for seven consecutive days. Animals in reference and control group received diclofenac sodium $(5 \mathrm{mg} / \mathrm{kg})$ and saline respectively. On the $8^{\text {th }}$ day of study the animals were anesthetized and sacrificed, and the pellets were dissected out carefully. Weight of the wet cotton pellets was noted. The weight of dry cotton pellets was measured after drying in an oven at $60^{\circ} \mathrm{C}$ for $24 \mathrm{~h}$. The mean weight of granuloma tissue formation around each pellet was calculated and the percentage inhibition was estimated using the following equation (Swingle,1972; Panthong, 2003, 2017).

$\%$ inhibition $=\frac{W_{c}-W_{t}}{W_{C}} \times 100$

Where $\mathrm{W}_{\mathrm{c}}=$ Weight of pellet in Control; $\mathrm{W}_{\mathrm{t}}=$ Weight of pellet in Test.

\section{Data analysis}

The in vitro results were expressed as the mean \pm SD of three parallel measurements. In vivo results were expressed as Mean \pm S.E.M. The difference between experimental groups were compared by ANOVA followed by Dunnett's test (Deepa et al., 2015).

\section{RESULT AND DISCUSSION \\ Evaluation of in vitro anti-inflammatory activity Denaturation of protein}

Denaturation of tissue protein is one of the well-reported causes of inflammation. In case of arthritis production of auto-antigen may occur due to denaturized of protein components. Prevention of protein denaturation can effectively reduce inflammation in such cases. Inhibition of heatinduced protein denaturation (stabilization of protein i.e. albumin) by NAR, NARNS has identified with the increment in absorbance of test samples with respect to control and reference drug Diclofenac sodium (Elisha et al., 2016). NARNS exhibited inhibition of protein denaturation of $96.05 \pm 3.84 \%$ at a concentration of $160 \mu \mathrm{g} / \mathrm{mL}$. which was significantly $(\mathrm{p}=0.48)$ greater than that of the drug diclofenac sodium and normal suspension of NAR ( $p=0.47)$. Increased ability of inhibiting the denaturation of structure of the protein albumin. This increased efficacy will be reducing the inflammation produced by the process of protein denaturation (Leelaprakash et al., 2011). This result showed that NARS might be more effective to inhibit protein denaturation in inflammatory process.

\section{Membrane stabilization method}

Released lysosomal enzymes in the process of inflammation produce several disorders. The extracellular activity of these enzymes is found to be associated with acute or chronic inflammation. Lysosomal membrane Stabilization is important in reducing inflammatory response. Lysosomal membrane Stabilization can be achieved by prevention of lysosomal constituent release by activated neutrophil which stimulate inflammation and damage of cells or tissues. 
Table I. Percentage inhibition of denaturation of protein albumin on NAR and NARNS.

\begin{tabular}{lcccccc}
\hline \multirow{2}{*}{ Compounds } & \multicolumn{4}{c}{ \% Inhibition of denaturation of protein } & IC50values \\
\cline { 2 - 6 } & $10 \mu \mathrm{g} / \mathrm{mL}$ & $20 \mu \mathrm{g} / \mathrm{mL}$ & $40 \mu \mathrm{g} / \mathrm{mL}$ & $80 \mu \mathrm{g} / \mathrm{mL}$ & $160 \mu \mathrm{g} / \mathrm{mL}$ & $(\boldsymbol{\mu g} / \mathbf{m L})$ \\
\hline Standard (Diclofenac & $19.98 \pm 1.98$ & $37.15 \pm 1.85$ & $57.52 \pm 0.11$ & $73.96 \pm 0.18$ & $83.03 \pm 2.49$ & $50.58 \pm 0.01$ \\
sodium) & $24.21 \pm 0.96$ & $41.14 \pm 2.46$ & $62.63 \pm 1.87$ & $79.82 \pm 4.78$ & $90.05 \pm 3.60$ & $37.90 \pm 0.22$ \\
NAR & $27.22 \pm 1.90$ & $45.28 \pm 2.26$ & $65.5 \pm 3.93$ & $82.63 \pm 4.95$ & $96.05 \pm 3.84$ & $29.75 \pm 0.24$ \\
NARNS &
\end{tabular}

Each value represents the mean $\pm \mathrm{SD}(\mathrm{N}=3)$

Table II. Percentage inhibition of hemolysis on NAR and NARNS.

\begin{tabular}{|c|c|c|c|c|c|c|}
\hline \multirow{2}{*}{ Compounds } & \multicolumn{5}{|c|}{ \% Inhibition of hemolysis } & \multirow{2}{*}{$\begin{array}{c}\text { IC50values } \\
(\mu \mathrm{g} / \mathrm{mL})\end{array}$} \\
\hline & $10 \mu \mathrm{g} / \mathrm{mL}$ & $20 \mu \mathrm{g} / \mathrm{mL}$ & $40 \mu \mathrm{g} / \mathrm{mL}$ & $80 \mu \mathrm{g} / \mathrm{mL}$ & $160 \mu \mathrm{g} / \mathrm{mL}$ & \\
\hline $\begin{array}{l}\text { Standard (Diclofenac } \\
\text { sodium) }\end{array}$ & $22.04 \pm 1.10$ & $32.03 \pm 1.92$ & $48.85 \pm 0.185$ & $54.24 \pm 2.70$ & $68.59 \pm 4.08$ & \\
\hline NAR & $27.3 \pm 1.35$ & $39.98 \pm 3.12$ & $56.27 \pm 3.36$ & & & \\
\hline NARNS & $34.65 \pm 1.36$ & $47.7 \pm 3.29$ & $61.3 \pm 5.49$ & $74.86 \pm 1.49$ & $84.77 \pm 1.69$ & $26.82 \pm 1.21$ \\
\hline
\end{tabular}

Each value represents the mean $\pm \mathrm{SD}(\mathrm{N}=3)$

Table III. Carrageenan induced paw edema on NAR and NARNS.

\begin{tabular}{lcccccc}
\hline \multirow{2}{*}{ Groups } & \multicolumn{5}{c}{ Paw thickness in (mm) } \\
\cline { 2 - 6 } & Before & $\mathbf{0 h}$ & $\mathbf{1 / 2 h}$ & $\mathbf{1 h}$ & $\mathbf{2 h}$ & $\mathbf{3 h}$ \\
\hline Carrageenan $(1 \% \mathrm{w} / \mathrm{v})$ & $1.83 \pm 0.10$ & $3.23 \pm 0.01$ & $3.93 \pm 0.01$ & $4.43 \pm 0.09$ & $5.16 \pm 0.14$ & $5.23 \pm 0.06$ \\
Carrageenan + & $1.8 \pm 0.07$ & $2.61 \pm 0.01$ & $3.1 \pm 0.02$ & $3.45 \pm 0.07$ & $4.01 \pm 0.09$ & $3.85 \pm 0.1$ \\
NARNS (50 mg/kg) & & & & & & \\
Carrageenan+Diclofenac & $1.84 \pm 0.12$ & $3.17 \pm 0.04^{* *}$ & $3.7 \pm 0.02^{* *}$ & $4.08 \pm 0.13^{* *}$ & $4.61 \pm 0.04^{* *}$ & $4.58 \pm 0.06^{* *}$ \\
Sodium (5 mg/kg) & & & & & & \\
Carrageenan + NAR & $1.8 \pm 0.03$ & $2.82 \pm 0.05^{* *}$ & $3.28 \pm 0.02^{* *}$ & $3.85 \pm 0.11^{*}$ & $4.38 \pm 0.04^{*}$ & $4.35 \pm 0.05^{*}$ \\
(50 mg/kg) & & & & & & \\
\hline
\end{tabular}

Observations are represented as Mean \pm SEM, $n=6$. Dunnett's test and ANOVA performed represent the significance of test.

The membrane stabilization property of NARNS was studied byanalyzing the inhibition of membrane lysis induced by hypotonicity in erythrocyte (Rao, 1989). The investigation revealed the NARNS significant ability in resisting the cell lysis in lesser concentrations as because of being lipophilic nanosuspension the formulation aides for easy absorption into cell through passive diffusion while compared to the standard drug diclofenac sodium and NAR. Compounds exhibiting membrane stabilizing activities are known for their ability to protect the cells from injurious substances and reduce the inflammatory mediator release triggered by phospholipases. From the study, it may be concluded that the NARNS has more membrane stabilizing property and exhibits antiinflammatory activity which is significantly greater compared to NAR and sodium $(\mathrm{P}=0.46)$ (Table I).
Evaluation of in-vivo anti-inflammatory activity Anti-inflammatory activity of NAR and NARNS by carrageenan induced paw edema model

Inflammation induced by carrageenan is acute with lesser systemic effects and reproducible. Induced paw edema is characterized by the release of several proinflammatory mediators such as cytokines, chemokines, histamines, serotonin, prostaglandins and oxygen-derived free radicals, increasing vascular permeability and cell migration mainly neutrophils (Singh et al., 2010). Carrageenan injection into the hind paw induces progressive edema which attains its maximum effectin $4 \mathrm{~h}$. In the case of negative control group, animals paw thickness was found at $(\mathrm{t}=0)$ was $3.23 \mathrm{~mm}$ and increased to $5.23 \pm 0.06 \mathrm{~mm}$ at $4 \mathrm{~h}$. Positive control (diclofenac) group animals had shown a decrease in paw thickness at $4 \mathrm{~h}$ which was significant compared to negative control group. 
Table IV. Percentage anti-inflammatory activity

\begin{tabular}{llll}
\hline \multirow{2}{*}{ Groups } & \multicolumn{3}{c}{ \% Anti-inflammatory activity } \\
\cline { 2 - 4 } & $1 \mathrm{~h}$ & $2 \mathrm{~h}$ & $4 \mathrm{~h}$ \\
\hline Carrageenan + NARNS $(50 \mathrm{mg} / \mathrm{kg})$ & 21.3 & 19.41 & 22.64 \\
Carrageenan + NAR(50 mg/kg) & 13.33 & 13.52 & 19.05 \\
Carrageenan + Diclofenac sodium $(5 \mathrm{mg} / \mathrm{kg})$ & 10.88 & 8.43 & 14.33 \\
\hline
\end{tabular}

Table V. Cotton pellet induced granuloma on NAR and NARNS.

\begin{tabular}{llccr}
\hline Groups & Wet weight & $\begin{array}{l}\text { \% inhibition } \\
\text { of wet weight }\end{array}$ & Dry weight & $\begin{array}{c}\text { \% inhibition } \\
\text { of dry weight }\end{array}$ \\
\hline Control(-ve) & $218.15 \pm 0.40$ & - & $58.3 \pm 0.26$ & \\
NARNS (40 mg/kg) & $147.5 \pm 0.25$ & $47.89 \%$ & $42.4 \pm 0.28$ & 26.58 \\
NAR (40 mg/kg) & $152.4 \pm 0.25^{* *}$ & $30.13 \%$ & $47.62 \pm 0.20^{* *}$ & 18.18 \\
Standard Diclofenac sodium $(5 \mathrm{mg} / \mathrm{kg})$ & $163.5 \pm 0.29^{* *}$ & $25.05 \%$ & $53.08 \pm 0.28^{* *}$ & 8.06 \\
\hline
\end{tabular}

All values are Mean \pm SEM, $n=6$. One-way Analysis of Variance (ANOVA) followed by Dunnett's test was performed as the test of significance.

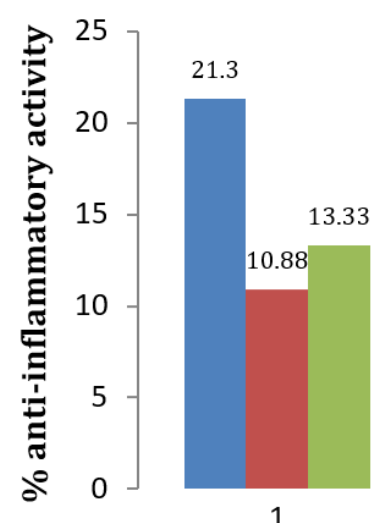

1

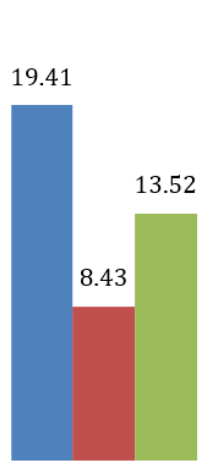

Time (hour)

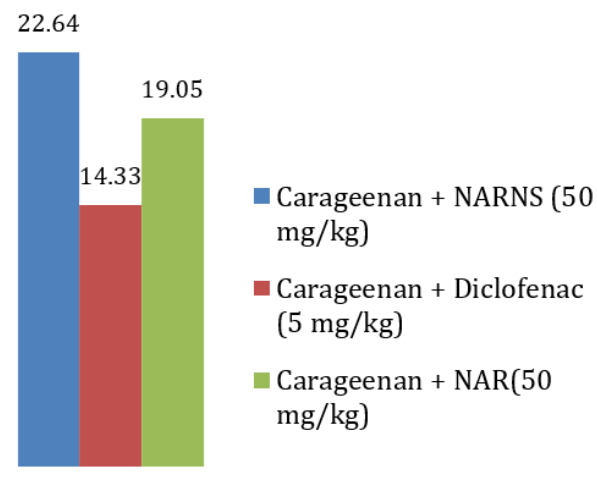

4

Figure 1. Representation of the percentage of inhibition of NAR and NARNS.

The paw thickness of NAR treated animals was found to be $(\mathrm{t}=0) \mathrm{mm} 2.82 \pm 0.05$ which was a mild increase compared to diclofenac sodium and the negative control group at $(\mathrm{t}=0)$. At the $3 \mathrm{~h}$, the paw thickness was $4.35 \pm 0.05$ for NAR which was a decrease from the $2 \mathrm{~h}$ paw thickness was $4.38 \pm 0.04 \mathrm{~mm}$ (Table III). NARNS treated animals were observed with a decrease in paw edema at the $3 \mathrm{~h}$ of the treatment. Statistically significant decrease in paw thickness $(\mathrm{p}<0.05)$ was demonstrated in NARNS treated group compared to the diclofenac sodium treated group. This decrease in paw edema may be an indication of inhibition of inflammation causing factors that causes paw edema while treated with carrageenan. The enhanced effect of NARNS may be due to the high systemic availability of the formulated product as it penetrates from Gastro-intestinal tract to systemic circulation easily due to lipophilicity and surface modification with P-gp efflux preventor. NARNS exhibited a significant anti-inflammatory activity compared to NAR and diclofenac sodium (Figure 1).

\section{Cotton pellet-induced granuloma}

Cotton pellet granuloma model is a study which indicates the proliferative phases of inflammation. Granuloma produced by the pellets is generally employed to measure the proliferation of chronic inflammation. Wet cotton pellet represents the transude material and the dry pellet weight corresponds the amount of granulomatous 


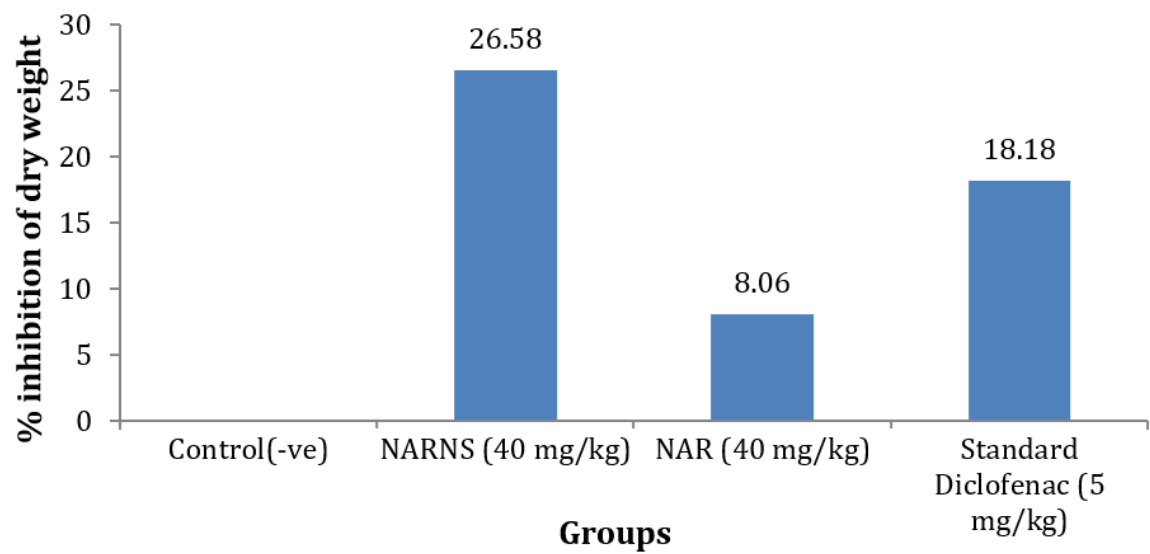

Figure 2. Representation of percentage of inhibition of NAR and NARNS.

tissue (Antonisamy et al., 2017). Edema and proliferative components in NARNS treated group were observed lesser compared to NAR and diclofenac sodium. The results revealed that the NAR and NARNS exhibited significantly lesser exudates and dry weight compared to diclofenac sodium $(\mathrm{p}<0.01)$ and the control group. NAR and NARNS showed $25.05 \%$ and $30.13 \%$ inhibition in weight of wet cotton pellets and $8.06 \%$ and $18.18 \%$ inhibition in weight of dry cotton pellets respectively data (Figure 2). It was assumed that the greater efficacy of NARS is due to high systemic availability and cellular penetration as particle size ranges in nanoscale and also lipid covering with surfactants (d- $\alpha$-tocopheryl polyethylene glycol 1000 succinate) of antieffluxion ability.

All values are Mean \pm SEM, $n=6$. One-way Analysis of Variance (ANOVA) followed by Dunnett's test was performed as the test of significance. This may be due to the strong free radical inhibitor or scavenger activity or acting possibly as primary oxidants of polyphenolic compounds such as flavonoids. The observations from the study revealed that the NARNS can be used as an effective anti-inflammatory compound which can be used in the treatment of several diseases including cancer, neurological disorder, aging, and inflammation. This study barn light on the efficacy of NARNS as an anti-inflammatory factor compared to NAR and Diclofenac sodium.

\section{CONCLUSION}

In vitro and in vivo study results indicate that the NAR possess anti-inflammatory property. Nanosuspension formulation is beneficial for NAR to increase the solvability and to protect from degradation. Conversion of NAR into NARNS enhanced anti-inflammatory activity of the drug in a lesser concentration compared to the normal NAR and diclofenac sodium. The NARNS inhibited the heat-induced albumin denaturation and stabilized the red blood cell membrane. Based on the in-vivo results, NARNS showed significantly good anti-inflammatory activity compared to diclofenac sodium.

\section{ACKNOWLEDGMENT}

We would like to say thank you to the JSS College of pharmacy for the supporting data

\section{REFERENCE}

Alhakmani F., 2013. Estimation of total phenolic content, in-vitro antioxidant and antiinflammatory activity of flowers of Moringaoleifera. Asian. Pac. J. Trop. Biomed. 3:623-627.

Ameer B., 1996. Flavanone absorption after naringin, hesperidin, and citrus administration. Clin.Pharmacol.Ther. 60:3440.

Antonisamy P., Agastian P., Kang CW., Kim NS., Kim JH., 2017. Anti-inflammatory activity of rhein isolated from the flowers of Cassia fistula L. and possible underlying mechanisms. Saudi J Biological Sciences. 28.

Cavia-Saiz M., 2010. Antioxidant properties, radical scavenging activity and biomolecule protection capacity of flavonoid NAR and its glycoside naringin: a comparative study. J. Sci. Food. Agri. 90:1238-1244.

Chathurdevi G., Umagowrie S., 2017. Potent bioactive metabolites of casuarina 
junghuhniana miq. Roots-a therapeutic approach. Int. J. Pharm. Sci. 9:158-165.

Choi JS., 1991. Antihyperlipidemic effect of flavonoids from Prunusdavidiana. J. Nat. Prod. 54: 218-224.

Dou W., 2009. Protective effect on NAR against experimental colitis via suppression of Tolllike receptor $4 / \mathrm{NF}-\mathrm{kB}$ signaling. Br.J.Nutr. 110(4):599- 608.

Elias G., 1988. Inhibition of albumin denaturation and anti-inflammatory activity of dehydrozingerone and its analogs. Indian. J. Exp. Biol. 26: 540-542.

Elisha IL, Dzoyem JP., McGaw J., Botha FS., Eloff JN. 2016. The anti-arthritic, anti-inflammatory, antioxidant activity and relationships with total phenolics and total flavonoids of nine South African plants used traditionally to treat arthritis. BMC Complementary and Alternative Medicine. 16:307.

Alhakmani F. 2014. Determination of total phenolic, in-vitro antioxidant and antiinflammatory activity of seeds and fruits of Zizyphusspina -Christi grown in Oman. Asian.Pac. J. Trop. Biomed. 4: 656-660.

Ferrero-Miliani L., 2007. Chronic inflammation: importance of NOD2 and NALP3 in interleukin-1beta generation. Clin. Exp. Immunol., 2: 147.

Gambhire M., 2008. Evaluation of antiinflammatory activity of methanol extract of BarleriaCristata leaves by in-vivo and in-vitro methods. The Internet J.Pharmacol. 7:21-27.

Gandhidasan R., 1991. Anti-inflamattory action of Laneacoromondelica by HRBC membranestabilisation. Fitotherapia. 62:8283.

Jain AK., 2011. The effect of the oral administration of polymeric nanoparticles on the efficacy and toxicity of tamoxifen. Biomaterials. 32:503-515.

James 0., 2009. In-vitro and in-vivo studies on the anti-oxidative activities, membrane stabilization and cytotoxicity of water spinach from ipogi ponds (Nigeria).Int. J.Pharmtech. Research. 1:474-482.

Jayaraman J., 2012. Anti-inflammatory role of naringenin in rats with ethanol induced liver injury. ToxicolMech Methods. 22:568576.

Leelaprakash G. and Dass SM. 2011. In vitro antiinflammatory activity of methanol extract of Enicostemmaaxillare. Int. J. Drug Dev. Res. 3(3):189-96.
Mizushima Y., Kobayashi M., 1968. Interaction of anti-inflammatory drugs with serum proteins, especially with some biologically active proteins. J Pharm Pharmacol. 20:16973.

Deepa M., Menon B., Darsanb and Ramalingam C. 2015. In vitro Evaluation Of The Antioxidant, Anti-Inflammatory And Antiproliferative Activities Of The Leaf Extracts Of Excoecaria AgallochaL .Int. J. Pharm Sci. 7:346-352.

Ntandou N G.., 2010. Analgesic and anti inflammatory effects of Cassia siamea Lam. Stem bark extracts. J. Ethnopharmacol. 127:108-111.

Panthong A., 2003. Anti-inflammatory and antipyretic properties of Clerodendrum petasites S. Moore. J. Ethnopharmacol. 85:151-156.

Rao AB., Sisodia P. and Sattur PB. 1989. Lysosomal membrane stabilization by antiinflammatory drugs. Indian journal of experimental biology. 27:1097-8.

Singh M., Kumar V., Singh I., Gauttam V. and Kalia AN., 2010. Anti-inflammatory activity of aqueous extract of Mirabilis jalapa Linn. leaves. Pharmacognosy research. 2(6):364.

Sumathi R., 2017. Formulation and evaluation of polymeric nanosuspension of NAR. Int. J. App. Pharm. 9:60-70.

Sumana Sarkhel., 2016. Evaluation of the antiinflammatory activities of Quillaja saponaria Mol. saponin extracts in mice. Toxicol. reports. 3:1-3.

Swingle KF., 1972. Phases of the inflammatory response to subcutaneous implantation of a cotton pellet and their modification by certain anti-inflammatory agents. J. Pharmacol. Exp. Ther. 183:226-234.

Varadarasu M., 2007. Evaluation of antiinflammatory and membrane stabilizing properties of ethanol extract of Cansjerarheedii J. Gmelin (Opiliaceae). Iranian J.Pharmacology and Therapeutics. 235-237.

Wen J., 2010. Preparation and physiochemical properties of the complex of NAR with hydroxypropyl-cyclodextrin. Molecules. 15:4401-4407.

William LS.,1989. The eicosanoids and their biochemical mechanism of action. Biochem J. 259:315-324.

Winter CA. and Risley GW., 1962. Nuss.Carrageenan-induced oedema in the hind paw of rat as an assay for anti- 
inflammatory activity. Proc. Soc. Exp. Biol.Ther. 111:544-547.

Wolfe MM. and David RL., 1999. Lichtenstein: Gastrointestinal toxicity of Non-steroidal anti-inflammatory drugs. $N$ Engl $\mathrm{J}$ Med. 340:1888-1899.
Yen FL., 2008. NAR-loaded nanoparticles improve the physicochemical properties and the hepatoprotective effect of NAR in orally administrated rats with CCl4-induced acute liver failure. Pharm Res. 26:893-902 